\title{
Maximising the efficiency of clinical screening programmes: balancing predictive genetic testing with a right not to know
}

\author{
Agnes G Schuurman ${ }^{\star, 1,4}$, Dorina M van der Kolk ${ }^{1,4}$, Marian A Verkerk², Erwin Birnie ${ }^{1}$, Adelita V Ranchor ${ }^{3}$, \\ Mirjam Plantinga ${ }^{1}$ and Irene $M$ van Langen ${ }^{1}$
}

We explored the dilemma between patients' right not to know their genetic status and the efficient use of health-care resources in the form of clinical cancer screening programmes. Currently, in the Netherlands, $50 \%$ risk carriers of heritable cancer syndromes who choose not to know their genetic status have access to the same screening programmes as proven mutation carriers. This implies an inefficient use of health-care resources, because half of this group will not carry the familial mutation. At the moment, only a small number of patients are involved; however, the expanding possibilities for genetic risk profiling means this issue must be addressed because of potentially adverse societal and financial impact. The trade-off between patients' right not to know their genetic status and efficient use of health-care resources was discussed in six focus groups with healthcare professionals and patients from three Dutch university hospitals. Professionals prefer patients to undergo a predictive DNA test as a prerequisite for entering cancer screening programmes. Professionals prioritise treating sick patients or proven mutation carriers over screening untested individuals. Participation in cancer screening programmes without prior DNA testing is, however, supported by most professionals, as testing is usually delayed and relatively few patients are involved at present. Reducing the number of $50 \%$ risk carriers undergoing screening is expected to be achieved by: offering more psychosocial support, explaining the iatrogenic risks of cancer screening, increasing out-of-pocket costs, and offering a less stringent screening programme for $50 \%$ risk carriers.

European Journal of Human Genetics (2015) 23, 1124-1128; doi:10.1038/ejhg.2014.269; published online 7 January 2015

\section{INTRODUCTION}

Should a medical professional refuse to perform clinical follow-up, as happened in the case of Sarah? Sarah is a 24-year-old woman from a family with Multiple Endocrine Neoplasia type 1 (MEN1) syndrome. Her sister and father carry a MEN1 mutation. Sarah therefore has a $50 \%$ risk of also carrying the familial mutation. She postponed a predictive DNA test and opted for clinical screening only, for which she was referred to an endocrinologist. He was willing to perform the recommended examinations (annual plasma evaluation of hormone profile, MRI and CT once every 2-3 years) only once and refused further screening because of the costs involved combined with his perceived lack of medical reason for screening of 50\% risk carriers. Although in some countries like the Netherlands ${ }^{1}$ and the United Kingdom $^{2}$ the national guidelines currently offer $50 \%$ risk carriers screening programmes identical to those for proven carriers, Sarah's endocrinologist felt that these guidelines could be questioned. After the clinical and radiological examinations, which showed no abnormalities, Sarah did request a DNA test, which showed she did not carry the familial mutation. Sarah's screening thus turned out to be unnecessary and health-care costs could have been avoided if her DNA test result had been known earlier.
Health-care costs have risen strongly over the past few years in many Western societies and there is no sign of any likely decline in the near future. There are increasing calls to reduce costs and avoid unnecessary care. ${ }^{3-6}$ Requiring patients to undergo predictive DNA testing and providing screening only to proven carriers would be more in line with the efficient use of health-care resources. However, where do we stand on the issues of patients' right not to know? How much importance should be given to patient autonomy compared with the efficient use of resources? Traditionally, the principle of autonomy as the right of self-determination is highly respected in the practice of clinical genetics, by which is meant the right of free choice and control of patients. Predictive DNA testing was implemented three decades ago for Huntington's disease, an incurable disease with no preventive or treatment options. As a consequence, counselors were reluctant to offer predictive testing and restricted the access to testing to only those counselees who were considered able to deal with the psychological burden of finding a mutation. In oncogenetics, the guidelines were written with the 'Huntington paradigm' as example, although some predictive measures were at hand and developed and improved over time and consequently health gains are now very well possible. Thus historically, the choice for or against predictive testing has always been a completely unconstrained one with even some tendencies to

${ }^{1}$ Department of Genetics, University of Groningen, University Medical Centre Groningen, Groningen, The Netherlands; ${ }^{2}$ Department of Internal Medicine, University of Groningen, University Medical Centre Groningen, Groningen, The Netherlands; ${ }^{3}$ Department of Health Psychology, University of Groningen, University Medical Centre Groningen, Groningen, The Netherlands

*Correspondence: AG Schuurman, Department of Genetics, University of Groningen, University Medical Centre Groningen, P0 Box 30001 , Groningen 9700 RB, The Netherlands. Tel: +31 50 3617148; Fax: +3150 3617231; E-mail: a.g.schuurman@umcg.nl

${ }^{4}$ These authors contributed equally to this work.

Received 6 May 2014; revised 2 September 2014; accepted 31 October 2014; published online 7 January 2015 
discourage the choice to know. Arguments against genetic testing were psychological and also motivated by fears of stigmatization and discrimination once a genetic predisposition to disease had been established. Therefore, genetic tests have always been different from other tests, such as cholesterol testing or blood pressure measurements that are offered routinely, although patients may decline the offer. Clinical geneticists have been aware of these differences and traditionally offer testing as a neutral choice, but other medical professionals might have been less aware of the reasons why testing is not routinely carried out.

Terms like costs or cost efficiency have only been mentioned rarely. ${ }^{7}$ What do health-care professionals and patients think about these issues? May costs be involved as an argument in offering or weighing genetic testing? In addition, do the stakeholders have suggestions on how to balance costs and the right not to know?

These issues will become even more relevant with the introduction of personal genome sequencing. Personal genome sequencing data can be used to construct genetic risk models that can distinguish individuals into categories of increased or reduced risk of cancer, for instance. ${ }^{8,9}$ According to their established risk, individuals could be offered a corresponding screening strategy. Here the same questions arise: may health professionals coerce people to first have their genetic status determined before assigning them to a corresponding screening strategy of preventive examinations? To obtain reasons for re-assessing the UK and Dutch genetic cancer screening guidelines, we set out to determine the opinions of health-care professionals and patients on this issue. We discussed the dilemma between the efficient use of health-care resources and respecting patients' right not to know in six focus groups composed of Dutch health-care professionals and patients.

\section{MATERIALS AND METHODS}

\section{Focus groups}

We held six focus group sessions in three university hospitals: Groningen (three professional groups and one patient group), Utrecht (one professional group), and Amsterdam (one professional group). The sessions were moderated by the same researchers and attended by 5-10 participants each. They were held between December 2011 and October 2012 and lasted about $90 \mathrm{~min}$.

\section{Participants}

For each hospital, purposive sampling was used and we invited all the clinical geneticists, genetic counsellors, and psychosocial workers involved in counselling patients with heritable cancer syndromes to attend. In the Netherlands, psychosocial workers are employed at clinical genetic departments and are frequently and intensively involved in the psychosocial care of patients who receive genetic counseling or who experience difficulty in making decisions about genetic testing. We further invited professionals involved in the screening of patients with heritable cancer syndromes, that is, gynaecologists, surgeons, nurse practitioners, endocrinologists, dermatologists, and gastroenterologists. We preferred heterogeneous groups because we anticipated that the diverse professional backgrounds and different screening programmes would stimulate the discussion. A total of 64 potential professional participants were sent an e-mail invitation explaining the aim of the study and the date for the focus group session in their own hospital.

Patients were selected from one university hospital and, at the time of their referral to the genetics department, had a 50\% risk of carrying a mutation for one of the following genetic cancer syndromes: hereditary breast and ovarian cancer, Lynch syndrome, multiple endocrine neoplasia type 1 (MEN1), or cutaneous malignant melanoma. A list of potential participants was compiled of patients who were known to have had difficulties in deciding whether to proceed with DNA testing. We excluded all patients with intellectual disabilities or psychological problems as stated in their medical record. After approval from the ethics committee of the University Medical Centre Groningen, 27 patients were sent a written invitation.

\section{Discussion guide and process}

The discussion guide was constructed after in-depth discussions between the research team members and covered the following themes: respecting the right not to know for autonomy, non-directiveness as a guiding principle in genetic counselling, screening programmes and their costs, and alternatives for reducing the costs of screening programmes and preventing overtreatment. Participants were asked to respond from their own daily practice and experience. Where appropriate, results from one focus group were used as input in other groups in order for the researchers to gain a better understanding of the themes and to explore the differences or agreements between the different groups. Three focus groups were moderated by DMvdK (Groningen, Utrecht), two by AGS (Groningen, Amsterdam), and one by MP (patient group, Groningen). DMvdK and AGS were note-takers in other instances and MP was the observer.

\section{Data analysis}

All the focus groups were audio-taped and verbatim transcripts were later produced and stored anonymously. Two researchers independently coded the transcripts (DMvdK and AGS) in Atlas.ti, a qualitative data analysis software package (version 5.7.1). They repeatedly read the transcripts and marked selected text passages with preliminary codes corresponding to the aim of the study. Disagreements between the researchers' coding were discussed until a consensus was reached. Later, the text under the defined codes was re-read and subthemes were discussed and assigned by all the research team members.

The Human Genome Variation Society recommends the term 'variation' instead of 'mutation'. However, the term mutation was used by both the professionals and the patients in our focus groups. For reasons of consistency and uniformity throughout the manuscript, we therefore decided to use the term mutation.

\section{RESULTS}

\section{Participants}

The number of participants in the five sessions for professionals varied between 6 and 10, with a total of 35 professionals (participation rate $35 / 64=55 \%$ ). The main reason for professionals not to participate was other obligations at the time of the group session.

The patient focus group had five participants (participation rate of $5 / 27=19 \%$ ). Three individuals did not know their DNA status at the time of the session. The other two knew they did not carry the familial mutation, but were known to have had difficulties in deciding whether to proceed with DNA testing. Most patients who declined to participate were unwilling to share their personal considerations with a group of strangers.

\section{The right not to know}

Patients' views of the right not to know. For patients, it is important that their right not to know is respected while still receiving access to cancer screening. There are several reasons why patients may not want to know their DNA status. One participant mentioned fear of stigmatisation and fear of being refused by insurance companies, if proven to be a mutation carrier. Another participant refused a DNA test because she anticipated that the parent who transmitted the mutation would feel guilty if she should prove to be a carrier. She said:

'I would have the idea that it would be a disappointment for my mother [if she, the daughter, is also shown to carry the familial BRCA mutation]. I would feel sorry for her.' [remark by a woman with unknown DNA status]

Several patients wanted screening even if they were not a carrier because it gave them a sense of reassurance. As proven non-carriers 
are not allowed to attend screening programmes, they saw it as an advantage to keep their DNA status as unknown.

'Even if you do not carry a BRCA mutation, as a woman you still have a $20 \%$ risk of getting breast cancer, so whether you're in the $20 \%$ or $80 \%$ risk group [of getting breast cancer], you always need to be alert. ... So I think I now have an advantage [in being screened twice a year] over women without a BRCA mutation who can just as easily get breast cancer.' [remark by a woman with unknown DNA status]

Family planning is another reason why people not want to know their DNA status. Two patients indicated that they wanted to have children and complete their family before being tested. Other reasons not to be tested were their unwillingness to undergo preventive surgical removal of the ovaries, or doubts about the operation, in the case of a proven BRCA mutation. Although predictive genetic testing does not require people to make such follow-up decisions about surgeries, people feel pressured to act upon the knowledge of having an increased risk. Therefore, patients sometimes experience a continuous process of making choices, which may need some time. Not only the wish to be screened, but also concerns about surgeries and family planning are playing a role in the complex process of decision making about predictive testing

'Well, yes, the consequences, what if I have the mutation? What am I going to do then? I'm not there yet, but I haven't any clear thoughts on that. I think like, if you know [you're a carrier], then you have to do something about it, you have to take action so to speak.' [remark by a woman with unknown DNA status]

Respecting the right not to know by professionals. Most health care professionals in our groups indicated that, over the last few years, their thoughts on the right not to know have shifted. They have become more directive to DNA testing. They mentioned that DNA testing has become more and more common and less controversial in the course of time, not only for professionals but also for patients. From a medical perspective, the professionals argued, the choice for predictive DNA testing in cancer syndromes before entering clinical screening is regarded as the most appropriate choice, since nowadays clinical screening and preventive surgery have proven to be good options when a mutation has been found. Health care professionals therefore emphasise the medical reasons for DNA testing. In addition, the professionals mentioned that it has become clear in the course of time that people can cope with the knowledge of being a mutation carrier of an oncogenetic disease.

Other reasons put forward by the professionals in favour of DNA testing were: clinical findings which cannot be interpreted easily with a patient's unknown DNA status, the wish to treat sick people or proven mutation carriers instead of preventing disease in healthy people, the risk of iatrogenic damage from screening methods, which can be avoided when DNA testing shows a person not to be a mutation carrier.

'Well, principally, my opinion is that if you can exclude [a mutation] reliably, which is the case with genetic analysis, you have to do that.' [remark by an endocrinologist]

Professionals are, nevertheless, willing to accept specific reasons for postponing a DNA test, such as fear of discrimination by insurance policies, or family or career planning. However, they are less willing to accept postponement for reasons that deal with psychological aspects, such as indecisiveness, or the wish, as mentioned above, to undergo screening (without prior DNA testing) in order to gain reassurance.

'I think we all agree it is undesirable [not to test] and, in the end, we all want a patient to take a DNA test. But the question is whether we should allow patients to wait until they're 60 years old? Or do you give them a year, or two or three, to get some things sorted in relation to certain affairs, children, taking out insurance, to get that done, and then to test.' [remark by a clinical geneticist]

In one university hospital the clinical geneticists and genetic counsellors were less explicit about this issue. They emphasized that the patient's autonomous choices should always be respected, regardless of the consequences. Psychosocial workers, who usually counsel patients who experience difficulty in making decisions, expressed their concerns about the complexity of decisions that patients have to make and the psychological burden of knowing to carry a mutation for a heritable cancer syndrome. They emphasized the importance of the current possibility for patients to participate in screening programmes without first having a DNA test.

'The choices you [as a patient] have to make are not easy. Because with all these possibilities and decisions, just because the options are available, you get into a process of making choices, and they involve decisions about things people don't really want to have to think about. And that can be an advantage, but it can also be a disadvantage. Because if you don't do anything, that's also a choice.' [remark by a psychosocial worker]

\section{Health-care costs}

Perceived responsibilities of physicians and patients in reducing healthcare costs. Professionals believe that decisions on health-care costs and the allocation of resources should, in the first place, be made by their representatives, insurance companies, and politicians.

'Well, in practice, looking at the health system in a broad sense, it isn't the individual doctors who determine these things; it's a kind of triage or budget agreements .... It's the [Dutch] insurance companies [who make agreements with hospitals].' [remark by a clinical geneticist]

Most of the professionals do not regard the health-care costs that go together with the screening of $50 \%$ risk carriers as a factor to be taken into account in their own daily practice. This can, in part, be explained by the small number of patients refusing a DNA test and the often temporary nature of the refusal. Therefore, the professionals currently feel no need to make choices based primarily on the costs of screening.

'So it's for a couple of years. That means one or two sets of medical examinations. What costs are we talking about then, altogether?' [remark by a genetic counsellor]

In addition, some of the professionals also argued that the Dutch health-care system currently promotes treatment over nontreatment. For example, a medical professional/gynaecologist gets reimbursed for performing surgery, but thoroughly talking with a patient about pros and cons of an operation is not reimbursed by the insurer. A gynaecologist said:

'We receive much more money [from the insurer] for performing an operation than for talking for an hour about the option not to operate.' [remark by a gynaecologist]

The professionals further argued that it is not the responsibility of an individual doctor or patient to decide which (expensive) interventions should be offered in specific cases. In fact, some even argued that emphasizing the cost of screening might force patients to undergo DNA testing or forego screening.

'Because I think if you were to say 'it does cost a lot and do consider it carefully', I mean, then you're already pushing people in a certain direction.' [remark by a psychosocial worker]

However, there are also patients who bring up the aspect of the treatment costs themselves.

'Now, I'm hearing it more and more, you see, I increasingly have patients who ask spontaneously what it costs and is it really useful, and should we test for this?' [remark by clinical geneticist] 
One patient said that she would consider it reasonable if professionals took the costs into account in deciding what clinical screening tests and in which frequency to offer to patients who were unwilling to have a DNA test. She would also agree to an obligation to be genetically tested before attending a screening programme. The other patients indicated that they felt no need to take the cost of treatment into account in deciding about DNA testing, as long as their healthcare costs were covered by their insurance policy. In their reasoning, some patients compared themselves to patients who incur health-care costs as a result of an unhealthy habit or lifestyle. They argue that having a heritable cancer syndrome in the family is something they cannot influence themselves, but are unfortunately confronted with. They should be allowed to make use of health care (and incur costs) in a screening programme without having to have a DNA test first, because the health-care costs of people who adopt an unhealthy habit or lifestyle are also covered by Dutch health insurance companies.

'Well, I think, like, I do some sports, I don't smoke, I don't drink, I haven't got diabetes, I'm not overweight, this [the screening tests for hereditary breast cancer] is the only cost I incur.' [remark by a woman with unknown DNA status]

Similar reasons were mentioned by some medical professionals, for example:

'We also treat people who smoke and people who are overweight, so these conditions are, in part, due to their own choices to be who they are.' [remark by a gastroenterologist]

Prioritising treatment. A number of professionals who are occupied with the performance of cancer screening expressed a preference towards prior DNA testing of a patient before starting screening. Their most important reason was the valuable time invested in 50\% risk carriers, half of whom will not carry the mutation. Treating sick patients or proven mutation carriers is considered more important than the screening of untested, apparently healthy, individuals.

'I mean that people who did not want a [DNA] test are using up time, energy, capacity and resources that can't then be used for people who did have a test or even for people who did have a test and became ill.' [remark by a surgeon]

In two university hospitals, several medical professionals said they would be inclined to refuse screening of $50 \%$ risk carriers and would try to convince them of the importance of a DNA test, but that they also felt obliged to accept the patient's wish not to be tested and to provide good care by following existing protocols.

'I don't want to examine healthy patients... If the consequence is that we have to examine all these healthy patients and perform imaging and biochemical tests, which is quite expensive, yes, I think that is unjustified.' [remark by an endocrinologist]

The medical professionals performing the screening programmes said to be directive by discussing the decision not to be tested each time an untested patient visits the hospital. The reluctance of the professionals to accept their choice not to be tested, was also mentioned by the patients.

'Yes, I did feel that I had to justify my decision. I had the idea that I would have needed to justify it less if I had decided to have the DNA test, that everyone would have found that more logical compared to this situation deciding not to know.' [remark by a woman with unknown DNA status]

Thus far, only few patients are refusing DNA testing while opting for screening. However, in the future, predictive genetic testing will be possible for a wide range of diseases, thus increasing the number of patients involved. According to the professionals, the problem of allowing more and more people into screening programmes without testing first will then become more urgent.

'And, of course, the more DNA tests that become possible, the more you're going to get this sort of thing, and that's why such an issue is relevant.' [remark by a clinical geneticist]

How to avoid unnecessary screening. While rejecting the possibility that patients should be coerced into undergoing genetic testing before cancer screening, other possibilities to prevent overtreatment and the inefficient use of health-care resources were considered. One of the suggestions was to invest more in the psychosocial support of such patients. Several professionals thought that psychosocial workers might be able to help them overcome their fears of being tested.

'And then it may well be cheaper to send the patient to a psychologist [or psychosocial worker] for a session, to discuss it [DNA testing] and then he might change his mind' [remark by a clinical geneticist]

Another suggestion from professionals to prevent overtreatment was to repeatedly clarify the medical disadvantages of ongoing clinical follow-up without a proven indication, such as chance findings or iatrogenic damage and other possibly adverse long-term health effects.

'The gastroenterologists also start putting up some resistance after a certain time when they have to check someone's colon for years, and they can of course perforate the colon during such a check-up.' [remark by a clinical geneticist]

Final suggestions to prevent overtreatment and inefficient use of health-care resources were to increase the out-of pocket (nonreimbursable) costs for $50 \%$ risk carriers and to offer them less intensive screening programmes.

'I think it could certainly involve charges and if you choose not to know but you want to have the check-ups, I can imagine that you, uhm, that you should have to pay for them' [remark by a woman with unknown DNA status].

'Or not doing an MRI every year but every other year - that would already make a huge difference.' [remark by a genetic counsellor].

\section{DISCUSSION}

To our knowledge, this is the first study to explore patients' right not to know their genetic status in relation to health-care costs in the rapidly developing field of genetics. There are several aspects to consider for our study and results. One strength of our study is that we included both health-care professionals and patients, resulting in a wide range of opinions that led to good discussions. We recorded no new insights in the last group session and appeared to have reached 'data saturation'. The one patient focus group was valuable in adding the patients' perspective. Unfortunately, not many patients were willing to participate in such sessions, but the opinions voiced by our patient group were recognised by the professionals and vice versa. In our study we focused only on a group of oncogenetic patients. Although some of the mentioned reasons for not wanting to know one's DNA status, such as the fear of genetic discrimination by insurance policies, ${ }^{10}$ lack of reassurance from negative test results, ${ }^{11,12}$ and concern about the psychological effects, ${ }^{13}$ have been described for other patient groups as well, future studies should investigate whether the reasons for temporarily accepting untested risk carriers for clinical screening also apply to other types of monogenetic and complex diseases. Further group discussions could also include policy-makers and health insurance company representatives.

The right not to know one's genetic status is a legitimate expression of patient autonomy. ${ }^{14-16}$ The health-care professionals participating in our study indicated that, over the last few years, their thoughts on patients' wishes not to know their DNA status have shifted. DNA 
testing has become more common and less controversial. In addition, nowadays clinical screening and preventive surgery have proven to be good options without severe psychological consequences. Therefore, health-care professionals are now more directive and try to motivate patients to choose for DNA testing. For some clinicians, the time they have to spend on looking after the 'worried well', while lacking enough time to care for the seriously ill, is a growing frustration. ${ }^{17}$ Some of the professionals in our groups also shared this frustration and wanted to exclude 50\% risk carriers from cancer screening. The Dutch genetic cancer screening guidelines currently oblige them to accept these patients. In order to reduce the number of participants without prior predictive testing in cancer screening programmes - and thereby increasing the efficient use of health-care resources - one patient and several professionals were in favour of introducing out-of-pocket costs for $50 \%$ risk carriers. Professionals also suggested that lowering the threshold for predictive testing could be achieved by increasing psychosocial support and better inform people about the iatrogenic risks of screening. Many professionals are not yet trained, nor supported, in talking to their patients about the need to avoid unnecessary care. ${ }^{18}$ Incorporating cost consciousness and good stewardship of resources into medical education and daily practice might help change this situation. ${ }^{19}$ Finally, more attention should be paid to the iatrogenic risks of cancer screening and to a less stringent screening programme that could be offered to $50 \%$ risk carriers. The Choosing Wisely approach, an initiative of the American Board of Internal Medicine (ABIM) Foundation, encourages physicians, patients and other health-care stakeholders to think and talk about medical tests and procedures that may be unnecessary. ${ }^{18,20}$ Future cancer screening guidelines regarding DNA testing may also draw upon the Choosing Wisely approach. The number of preventable diseases for which risk predictions can be made by DNA testing or genome sequencing is rising rapidly, thereby increasing the dilemma between respecting the right not to know and the efficient use of health-care resources.

In summary, health-care professionals prioritise treating sick patients or proven mutation carriers over the screening of untested, apparently healthy, individuals and therefore prefer potential carriers to undergo a DNA test as a prerequisite for cancer screening. Participation in clinical cancer screening programmes without prior DNA testing is, however, respected by most health-care professionals, especially as genetic testing is usually only delayed temporarily and only few patients are involved yet. Possibilities to reduce the number of $50 \%$ risk carriers undergoing repeated cancer screening are: offering more psychosocial support, fully explaining the iatrogenic risks of cancer screening, increasing out-of-pocket costs, and offering a less stringent screening programme for $50 \%$ risk carriers.

Professionals and patients in our study have proposed different measures to reduce the number of $50 \%$ risk carriers participating in clinical screening programmes. More research is needed to gain a better understanding of their impact on patient autonomy, the efficient use of resources, and the prevention of unnecessary screening. This evidence, together with a broader discussion between different stakeholders on preventing overtreatment and the inefficient use of health-care resources in cancer screening, while respecting patients' right not to know, forms necessary input for re-assessing the genetic cancer screening guidelines.

\section{CONFLICT OF INTEREST}

The authors declare no conflict of interest.

\section{ACKNOWLEDGEMENTS}

We thank all patients and professionals for participating in the focus group discussions and Jackie Senior for editing the manuscript.

1 Brekelmans CT, Seynaeve C, Bartels CC et al: Effectiveness of breast cancer surveillance in BRCA1/2 gene mutation carriers and women with high familial risk. J Clin Oncol. 2001; 19: 924-930.

2 National Institute for Healt and Care Excellence (NICE): Familial breast cancer: Classification and care of people at risk of familial breast cancer and management of breast cancer and related risks in people with a family history of breast cancer 2013, http://publications.nice.org.uk/familial-breast-cancer-cg164.

3 Malach M, Baumol WJ: Further opportunities for cost reduction of medical care. J Community Health 2010; 35: 561-571.

4 Lenzer J: Unnecessary care: are doctors in denial and is profit driven healthcare to blame? Br Med J 2012; 345: e6230.

5 Huckman RS, Kelley MA: Public reporting, consumerism, and patient empowerment. N Engl J Med 2013; 369: 1875-1877.

6 Moynihan R, Doust J, Henry D: Preventing overdiagnosis: how to stop harming the healthy. Br Med J 2012; 344: e3502.

7 Berliner JL, Fay AM, Cummings SA, Burnett B, Tillmanns T: NSGC practice guideline: risk assessment and genetic counseling for hereditary breast and ovarian cancer. J Genet Couns 2013; 22: 155-163.

8 Khoury MJ, Janssens AC, Ransohoff DF: How can polygenic inheritance be used in population screening for common diseases? Genet Med 2013; 15: 437-443.

9 Chowdhury S, Dent T, Pashayan N et al: Incorporating genomics into breast and prostate cancer screening: assessing the implications. Genet Med 2013; 15: 423-432.

10 Henneman L, Van Hoyweghen I Moving beyond public fear of genetic discrimination 2013, https://www.genevaassociation.org/media/77290/ga2013-health28-hennemanvan_hoyweghen.pdf.

11 Halbert $\mathrm{CH}$, Lynch $\mathrm{H}$, Lynch J et al: Colon cancer screening practices following genetic testing for hereditary nonpolyposis colon cancer (HNPCC) mutations. Arch Intern Med 2004; 164: 1881-1887.

12 Michie S, Smith JA, Senior V, Marteau TM: Understanding why negative genetic test results sometimes fail to reassure. Am J Med Genet A 2003; 119A: 340-347.

13 Godard B, Pratte A, Dumont M, Simard-Lebrun A, Simard J: Factors associated with an individual's decision to withdraw from genetic testing for breast and ovarian cancer susceptibility: implications for counseling. Genet Test 2007; 11: 45-54.

14 Andorno R: The right not to know: an autonomy based approach. J Med Ethics 2004; 30: 435-439.

15 Bortolotti L, Widdows $\mathrm{H}$ : The right not to know: the case of psychiatric disorders. J Med Ethics 2011; 37: 673-676.

16 Herring J, Foster C: 'Please don't tell me'. The right not to know. Camb Q Healthc Ethics 2012; 21: 20-29.

17 Glasziou P, Moynihan R, Richards T, Godlee F: Too much medicine; too little care. BMJ 2013; 347: f4247.

18 Reuben DB, Cassel CK: Physician stewardship of health care in an era of finite resources. JAMA 2011; 306: 430-431.

19 Rosenbaum L, Lamas D: Cents and sensitivity-teaching physicians to think about costs N Engl J Med 2012; 367: 99-101.

20 ABIM Foundation: Choosing wisely 2014, http://www.choosingwisely.org/.

(2) (i) $\odot$ This work is licensed under a Creative Commons Attribution-NonCommercial-NoDerivs 3.0 Unported License. The images or other third party material in this article are included in the article's Creative Commons license, unless indicated otherwise in the credit line; if the material is not included under the Creative Commons license, users will need to obtain permission from the license holder to reproduce the material. To view a copy of this license, visit http://creativecommons.org/licenses/by-nc-nd/3.0/ 